\title{
Impact of the combination of an angiotensin II receptor blocker and low-dose hydrochlorothiazide on patients with morning hypertension
}

\author{
Xian Wu Cheng and Toyoaki Murohara \\ Hypertension Research (2012) 35, 702-703; doi:10.1038/hr.2012.57; published online 26 April 2012
}

$\mathrm{E}^{\mathrm{p}}$ pidemiological studies have shown the existence of a circadian pattern in the onset of adverse cardiovascular (CV) events, which occur most frequently in the morning (0600 hours to noon). ${ }^{1,2}$ The mechanisms involved in the morning increase in $\mathrm{CV}$ events are not well understood. However, Kario et al. $^{3}$ have shown an association between the CV events and morning blood pressure (BP) surge. For example, the incidence of stroke in the morning hours was shown to be significantly increased in those with an exaggerated morning BP surge compared with those without. ${ }^{3}$

Renin-angiotensin system (RAS) inhibitors (angiotensin-converting enzyme inhibitor or angiotensin II receptor blockers) are widely recommended as first-line drugs to prevent target organ damage in hypertensive patients at high risk of CV events; however, it is often difficult to achieve the target BP by treatment solely with RAS inhibitors. Recently, the American Society of Hypertension and the European Society of Hypertension recommended the use of a specific combination of agents that elicit different antipressor mechanisms or that block counterregulatory responses, to further reduce $\mathrm{BP}$ and to increase the number of patients achieving the BP treatment goal. In addition, in many cases, drug adherence can be enhanced through the use of a combination tablet that contains optimal doses of two or three different agents.

Therefore, one of the most intriguing questions in clinical practice for hypertension

XW Cheng and T Murohara are at the Department of Cardiology, Nagoya University Graduate School of Medicine, Nagoya, Japan.

E-mail: murohara@med.nagoya-u.ac.jp is determining which combinations and doses of drugs are ideal additions to the first-line RAS inhibitors. BP reduction with a diuretic-based regimen has been shown to decrease the incidence of stroke, heart failure and other CV events. ${ }^{4}$ Most of these studies used chlorthalidone, a thiazide-class diuretic; yet, 95\% of thiazide prescriptions are for hydrochlorothiazide (HCTZ). ${ }^{5}$ Previous studies have documented that the combination of losartan $(50 \mathrm{mg})$ with lowdose HCTZ (12.5 mg) successfully reduced BP in patients with essential hypertension. ${ }^{6,7}$ However, there has been no study that tested the hypothesis that selective suppression of an exaggerated morning BP surge leads to a regression of target organ damage and a reduction in subsequent $\mathrm{CV}$ events.

In this issue of Hypertension Research, Ueda et al. ${ }^{8}$ reported an interesting clinical study, in which they compared the efficacy and safety of a losartan $(50 \mathrm{mg}) / \mathrm{HCTZ}$ $(12.5 \mathrm{mg})$ combination and high-dose losartan $(100 \mathrm{mg})$ in patients with morning hypertension or a morning BP surge. ${ }^{9}$ Combination therapy induced a greater reduction in the morning systolic $\mathrm{BP}$ than did high-dose losartan therapy $(131.5 \pm 11.5$ vs. $142.5 \pm 13.6 \mathrm{~mm} \mathrm{Hg}, \quad P<0.001)$. As a result, ARB/HCTZ combination therapy had a twofold greater rate of achieving the target morning BP than did high-dose losartan therapy.

Recent studies have shown that the addition of low-dose HCTZ (12.5 mg) to losartan (50 mg) once daily significantly decreased the urinary protein/creatinine ratio in hypertensive patients with chronic kidney disease without changing the serum creatinine level and estimated glomerular filtration rate. ${ }^{10}$
Ueda et al. also confirmed and reported that this combination therapy significantly improved the urine albumin/creatinine ratio in patients with morning hypertension. ${ }^{9}$ It should be noted that daily administration of high-dose losartan (100 mg) had no significant effect on urinary albumin levels. Ueda et al. propose that the reduction in the urinary protein/creatinine ratio might be attributable to the greater BP-lowering effect of the combination therapy. It would be interesting to investigate whether ARB/ HCTZ combination therapy can reduce the urinary albumin excretion ratio more intensively than high-dose ARB in patients with similar BP levels.

The present study clearly shows that the combination of two drugs with different antipressor efficacies, namely $\mathrm{ARB}$ and HCTZ, was superior to high-dose ARB monotherapy in terms of reducing the BP and the renal damage in patients with morning hypertension. The combination of ARB and HCTZ elicits a synergistic BP reduction through complementary mechanisms. ${ }^{11}$ Furthermore, the current study was performed in Japan, and Japanese hypertensive patients have a nature of saltinduced hypertension compared with Caucasians. ${ }^{12}$ In this regard, HCTZ might increased nocturnal diuresis during asleep, and this might have a key role in the further reduction of morning $\mathrm{BP}$.

There are several limitations to this study. First, the authors evaluated only the systolic BP. A recent study has indicated that there is a so-called J-curve phenomenon in terms of the relationship between the diastolic BP and coronary events. ${ }^{13}$ Although the combination of losartan and HCTZ significantly reduced 


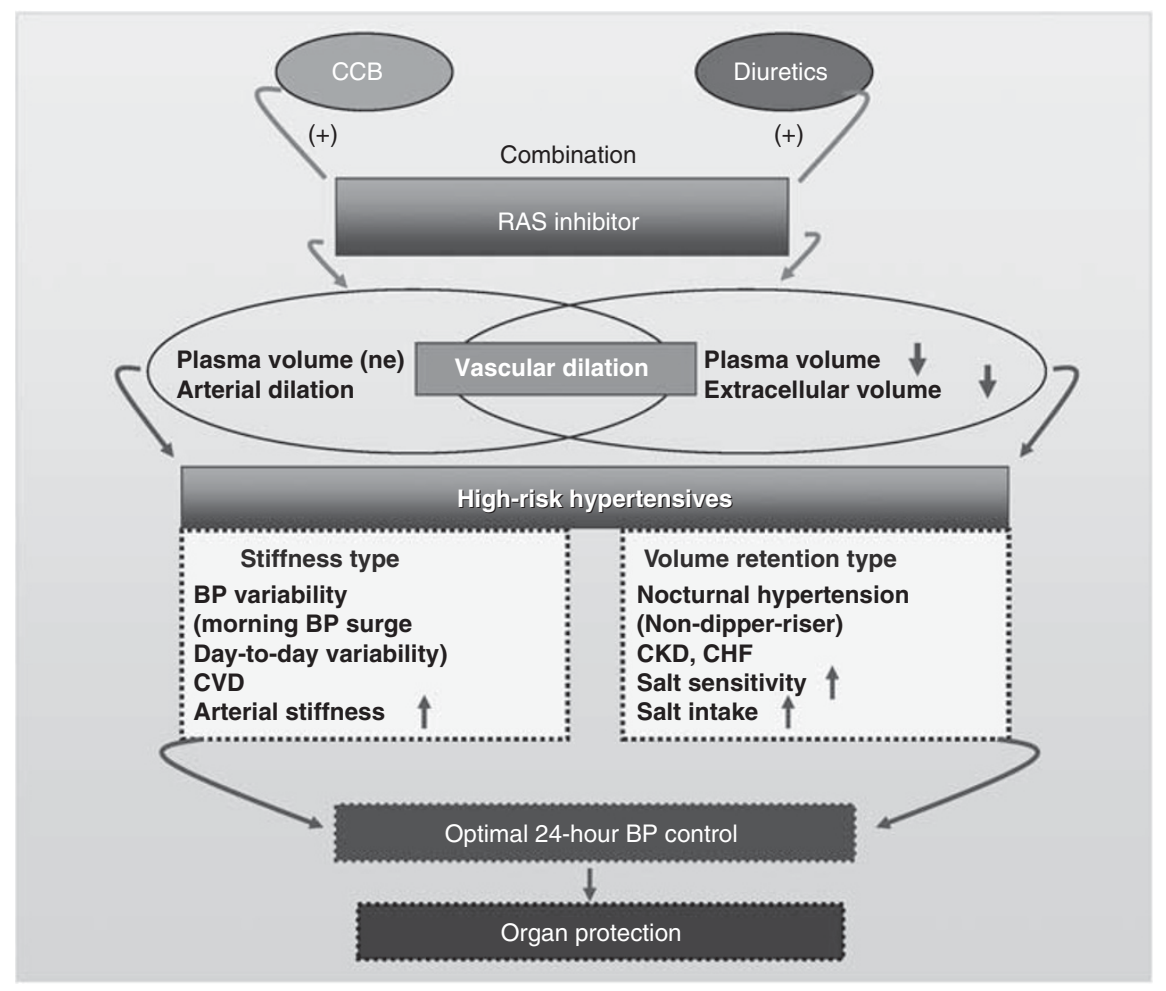

Figure 1 Proposed RAS-inhibitor-based combination therapy with diuretics or CCBs for hypertensive patients at high risk. CVD, cardiovascular disease; CKD, chronic kidney disease; CHF, congestive heart failure; ne, no effect. A full color version of this figure is available at Hypertension Research online.

the systolic BP and renal damage in this study, a strong reduction of the diastolic BP may worsen coronary events. Second, the efficacy of a combination of an ARB and a calcium channel blocker (CCB) on the morning BP surge was not tested in the present study. It should be noted that the ARB/CCB regimen is another strong BP modifier and CV event inhibitor. Thus, in the future, it would be interesting to compare the $\mathrm{ARB} / \mathrm{HCTZ}$ and ARB/CCB regimens for patients with a morning BP surge (Figure 1).

Finally, despite several limitations, this study is of great interest to the scientific community because it demonstrates clearly for the first time that, in patients with morning hypertension, the combination of losartan (50 mg) and low-dose HCTZ $(12.5 \mathrm{mg})$ once daily resulted in a greater reduction in BP (including morning, evening and clinic BP) without any diureticrelated adverse effects than did high-dose losartan (100 mg). A larger-scale, long-term, randomized trial is warranted to address whether this combination therapy would result in a greater reduction in future $\mathrm{CV}$ events in patients with a morning $\mathrm{BP}$ surge.
1 Muller JE, Tofler GH, Stone PH. Circadian variation and triggers of onset of acute cardiovascular disease. Circulation 1989; 79: 733-743.

2 White WB. Cardiovascular risk and therapeutic intervention for the early morning surge in blood pressure and heart rate. Blood Press Monit 2001; 6: 63-72.

3 Kario K, Pickering TG, Umeda Y, Hoshide S, Hoshide $Y$, Morinari M, Murata M, Kuroda T, Schwartz JE, Shimada K. Morning surge in blood pressure as a predictor of silent and clinical cerebrovascular disease in elderly hypertensives: a prospective study. Circulation 2003; 107: 1401-1406.

4 Ernst ME, Moser M. Use of diuretics in patients with hypertension. N Engl J Med 2009; 361: 2153-2164.

5 Ernst ME, Neaton JD, Grimm Jr RH, Collins G, Thomas W, Soliman EZ, Prineas RJ. Long-term effects of chlorthalidone versus hydrochlorothiazide on electrocardiographic left ventricular hypertrophy in the multiple risk factor intervention trial. Hypertension 2011; 58: 1001-1007.

6 Minami J, Abe C, Akashiba A, Takahashi T, Kameda T, Ishimitsu T, Matsuoka H. Long-term efficacy of combination therapy with losartan and low-dose hydrochlorothiazide in patients with uncontrolled hypertension. Int Heart J 2007; 48: 177-186.

7 Hamada T, Mizuta E, Kondo T, Hirai M, Yamada K, Kato $M$, Shigemasa $C$, Yamamoto $Y$, Ninomiya $H$, Igawa $O$, Hisatome I. Effects of a low-dose antihypertensive diuretic in combination with losartan, telmisartan, or candesartan on serum urate levels in hypertensive patients. Arzneimittelforschung 2010; 60: 71-75.

8 Ueda T, Kai H, Imaizumi T, on behalf of the MAPPY Study Investigators. Losartan/hydrochlorothiazide combination vs. high-dose losartan in patients with morning hypertension - a prospective, randomized, open-labeled, parallel-group, multicenter trial. Hypertens Res 2012; 35: 708-714.

9 Harada K, Izawa H, Nishizawa T, Hirashiki A, Murase $Y$, Kobayashi M, Isobe S, Cheng XW, Noda A, Nagata K, Yokota M, Murohara T. Beneficial effects of torasemide on systolic wall stress and sympathetic nervous activity in asymptomatic or mildly symptomatic patients with heart failure: comparison with azosemide. J Cardiovasc Pharmacol 2009; 53: 468-473.

10 Abe M, Okada K, Maruyama T, Matsumoto K. Renoprotect and blood pressure lowering effect of low-dose hydrochlorothiazide added to intensive renin-angiotensin inhibition in hypertensive patients with chronic kidney disease. Int J Clin Pharmacol Ther 2009; 47: 525-532.

11 Mackay JH, Arcuri KE, Goldberg AI, Snapinn SM, Sweet CS. Losartan and low-dose hydrochlorothiazide in patients with essential hypertension. A double-blind, placebo-controlled trial of concomitant administration compared with individual components. Arch Intern Med 1996; 156: 278-285.

12 Kimura G. Kidney and circadian blood pressure rhythm. Hypertension 2008; 51: 827-828.

13 Messerli FH, Panjrath GS. The J-curve between blood pressure and coronary artery disease or essential hypertension: exactly how essential? J Am Coll Cardiol 2009; 54: 1827-1834. 